drug errors have been reduced. However this could be due to a number of factors and ongoing data collection is required to identify the trend. The SPG has now been disseminated to Sue Ryder Hospices nationally.

\section{ASSESSING THE IMPACT OF A ONE DAY ADVANCED COMMUNICATION SKILLS COURSE FOR QUALIFIED AND UNQUALIFIED NURSES IN A HOSPICE SETTING}

L Datta-Paulin, S Salt. Trinity Hospice, Blackpool

10.1136/bmjspcare-2019-ASP.36

Background Trinity Hospice, Blackpool run an internal advanced communications course for qualified and unqualified nursing staff working across inpatient and community settings. Groups of up to 8 participants undertake role play with a facilitator and an actor, using commonly encountered communication scenarios. This project assessed the impact of the course on participants' confidence in communicating in difficult situations and the emotional burden associated with undertaking the course.

Methods Pre- and post-course questionnaires were filled in by all participants, and then retrospectively analysed.

Results 45 people were trained over 18 months, including 27 qualified and 18 unqualified staff-members. 1 questionnaire was incompletely filled out giving a total sample of 44 .

$84 \%$ of participants described negative emotions (e.g. nervous, unsure) before the course.

$82 \%$ described positive emotions (e.g. relaxed, confident, energised) after the course.

$50 \%$ of participants showed improvement in confidence in a challenging conversation with a patient or relatives

$68 \%$ showed improvement in confidence in a challenging conversation with a colleague.

$50 \%$ showed improvement in confidence in handling strong emotions.

$34 \%$ showed improvement in confidence in identifying a patient or relative's concerns.

$59 \%$ showed improvement in confidence in challenging problematic behaviour by a patient or relative.

$98 \%$ of the participants reported that the course had met their needs, and scored the course as being interesting, informative, useful, enjoyable, respectful and safe.

Conclusion The subjective response to the course was positive, with a shift from negative to positive emotional responses. At least half of participants improved in all but one area of communication. We feel this project supports the validity of the advanced communication skills course for development of confidence in challenging areas of communication, for both qualified and unqualified nursing staff.

\section{$14 \quad$ PHASE OF ILLNESS SURVEY}

Joanna Vriens. Phyllis Tuckwell Hospice Care

10.1136/bmjspcare-2019-ASP.37

Background Phase of Illness (POI) is one of the measures in the Outcome Assessment and Complexity Collaborative (OACC) suite of measures, and describes the current stage in the patient's illness according to the care needs of the patient and their family. The phases are: stable, unstable, deteriorating, dying and deceased. POI is documented at every patient clinical contact by a range of healthcare professionals (HCPs); therefore consistency of phase assessment is important. The aim of this survey was to assess the consistency of POI assessment.

Method A survey was emailed to all clinical staff and had two peer reviewed fictional case studies evolving over time. They were asked to select the POI that best described the patient's situation at various time points.

Results Fifty-one HCPs completed scenario 1 and 41 completed scenario 2. The range of HCPs included nurses, doctors, physiotherapists, occupational therapists, counsellors, social workers and complementary therapists.

Scenario 1 - Mrs A with metastatic lung cancer, and six phase assessments. There was majority agreement across all phase assessments. The phase assessment with least agreement was Q5 stable 63\%; unstable 1\%; deteriorating 35\%. All other assessments had $>70 \%$ agreement with a particular POI.

Scenario 2 - Mr B with Motor Neurone Disease (MND), and seven phase assessments. There was less agreement across the phase assessments. Three POI assessments had <70\% majority agreement: Q3- Stable 10\%, Unstable 45\%, Deteriorating 45\%; Q4 - Stable 68\%, Unstable 7.5\%, Deteriorating 24.5\%; Q7 - Stable 7.5\%, Unstable 55\%, Deteriorating $37.5 \%$.

Conclusion There was less consistency of POI assessment in the MND patient scenario, particularly in terms of unstable and deteriorating phases, compared to the cancer patient scenario. This disparity may reflect the different disease trajectories and HCP familiarity with each condition. This has led to further multidisciplinary team training focusing on POI assessment in non-cancer conditions.

\section{EXPLORING THE USE OF INNOVATIVE TECHNOLOGY TO DELIVER JUNIOR DOCTORS INDUCTION AT THE HOSPICE}

Siwan Seaman, Rebecca Evans, Mark Taubert. Marie Curie Hospice Cardiff and the Vale, Cardiff University, Velindre University NHS Trust

\subsection{6/bmispcare-2019-ASP.38}

Background There is a paucity of published evidence evaluating the effectiveness of induction methods for trainee doctors. In any 12 month period 17 trainees (3 foundation doctors, 8 core medical trainees and 6 specialty trainees) will rotate through our hospice, resulting in the medical induction programme being repeated at least 8 times per year.

Methods Considering prudent healthcare principles, the aim was to evaluate the current induction programme and to scope alternative, more efficient delivery methods. Feedback on the induction of 16 trainees was analysed in addition to feedback from 29 medical students who had experienced virtual reality (VR) palliative care teaching methods locally as well as an online survey sent to 33 previous trainees at the hospice to ascertain their views on alternative delivery methods.

Results Amongst the cohort who had experienced VR, the feedback was generally positive. There was however a general reluctance to consider new techniques amongst past trainees of the hospice who, unanimously, valued the face-to-face induction they had received, this is consistent with positive GMC trainee survey responses at the hospice to the induction question. 